\title{
Intermittent positional axillary vein compression: a rare complication of CIED implantation
}

\author{
Roman Steckiewicz' (D), Przemysław Stolarz², Elżbieta Świętoń \\ 'Department of Cardiology, Central University Hospital in Warsaw, Poland \\ ${ }^{2}{ }_{1}^{\text {st }}$ Department of Cardiology, Medical University of Warsaw, Poland
}

\begin{abstract}
An 81 -year-old male with an implanted DDD pacemaker reported fluctuating edema accompanied by pain and paresthesia affecting the hand and arm on the side the pacemaker had been implanted. These symptoms, which developed several weeks after the procedure, were more pronounced in the morning and resolved during the day. Venography showed axillary vein compression, whose severity varied with different arm positions. Changing the location of the pacemaker pocket restored normal venous blood flow at the site of compression and relieved the patient's symptoms, which was confirmed during follow-up.
\end{abstract}

Key words: pacemaker, venography, axillary vein, venous compression, occlusion

Acta Angiol 202I; 27, 4: 144-146

\section{Introduction}

In most cardiac implantable electronic device (CIED) implantation procedures involving the intravenous lead placement, the body of the device is placed in a subcutaneous pocket created in the infraclavicular region anterior to the pectoralis muscles.

Postoperatively, some devices migrate with respect to their original placement within the pre-pectoral pocket. In such cases, depending on the direction and extent of its migration, the device may compress nearby anatomical structures, which may produce symptoms. CIED migration toward the axillary fossa may produce vascular and/or neurological manifestations.

Vascular manifestations are typically due to axillary vein compression despite the proximity of both axillary artery and vein in this region. This is due to the different anatomical structures of arterial and venous walls, with the latter more readily deformed by pressure. Potential neurological symptoms correspond to the area innervated by the axillary nerve, which is the main branch of the brachial plexus. Similar symptoms may also manifest in other diseases, which must be considered in the differential diagnosis [1-4].

\section{Case report}

An 81 -year-old male with an implanted DDD-mode cardiac pacemaker was hospitalized due to fluctuating edema accompanied by episodes of pain in the hand and arm on the side the pacemaker had been implanted. These symptoms developed a few weeks after pacemaker implantation, were most pronounced in the morning, and gradually resolved during the day. Venography, which was performed in a supine position with the arms along the torso, revealed the axillary vein compressed by the pacemaker (Fig. IB).

This local obstruction in the flow of contrast improved when the device was moved medially towards the sternum (Fig. 2A) and when the patient changed the position of his arm, for example by lifting it (Fig. 2B).

A steady, normal flow of contrast through the previously compressed vein was achieved following a surgical repositioning of the pacemaker pocket medially. 

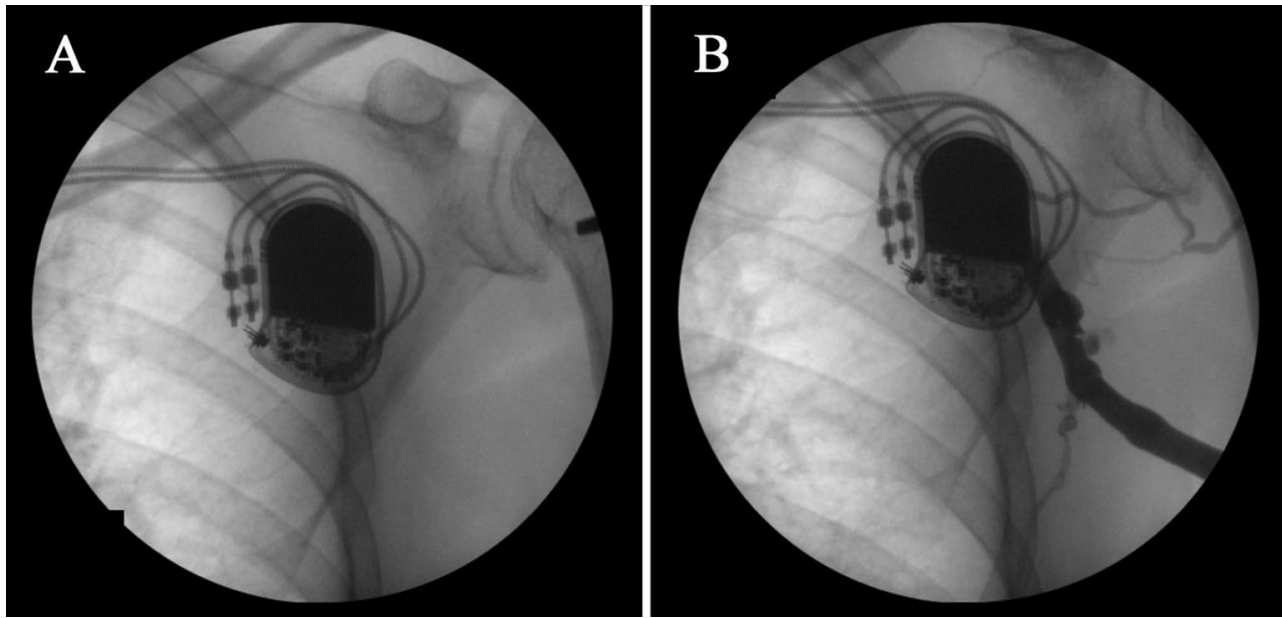

Figure I. An 8I-year-old male with an implanted DDD-mode cardiac pacemaker (A). Radiographic overview of the left clavipectoral triangle, with the pacemaker in its original position (B). A view of the same location shows the mechanism behind the obstructed flow of contrast
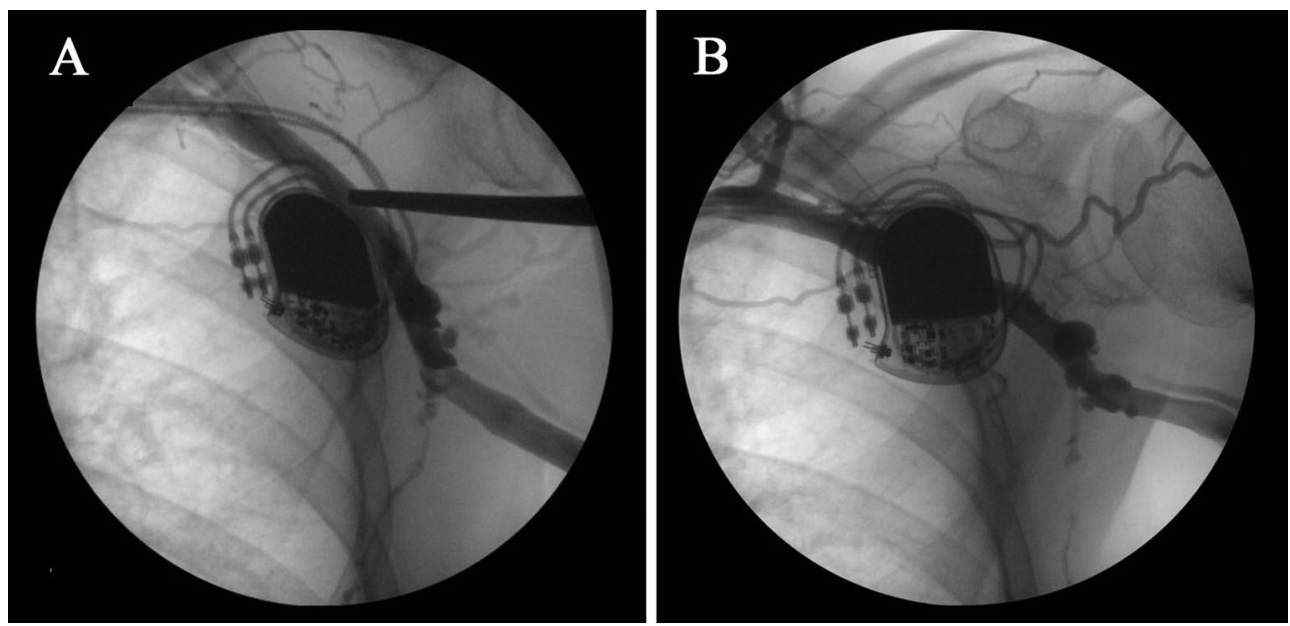

Figure 2. Normal flow of contrast in response to changes in the spatial relation between the pacemaker and the vein (Fig. I). The arm is adducted, and the pacemaker has been moved medially $(\mathbf{A})$. The arm is abducted and raised (B)

Follow-up venography images revealed post-thrombotic lesions in the subclavian vein (Fig. 3B). The resulting subclavian vein obstruction was clearly apparent with the arm slightly lowered when the flow of contrast became redirected via collateral circulation (Fig. 3B), whereas limb elevation yielded a normal flow of contrast through the subclavian vein, which seemed to suggest an absence of post-thrombotic lesions (Fig. 2B).

By showing a complete resolution of the patient's symptoms, outpatient follow-up assessments confirmed the causal relationship between the previous position of the pacemaker and the symptoms of the axillary nerve and axillary artery suppression. At their current stage, the post-thrombotic lesions incidentally found in the subclavian vein could not have been responsible for the clinical symptoms presented above.

\section{Discussion}

The number of CIED implantation procedures involving intravenous lead placement via the clavipectoral triangle has been systematically increasing, thus resulting in a growing number of cases where the pocket for the implanted device is created in the infraclavicular region.

Despite the progressive miniaturization of electrotherapy devices, spontaneous migration of the implanted device may occur (as a result of a loosely structured, 

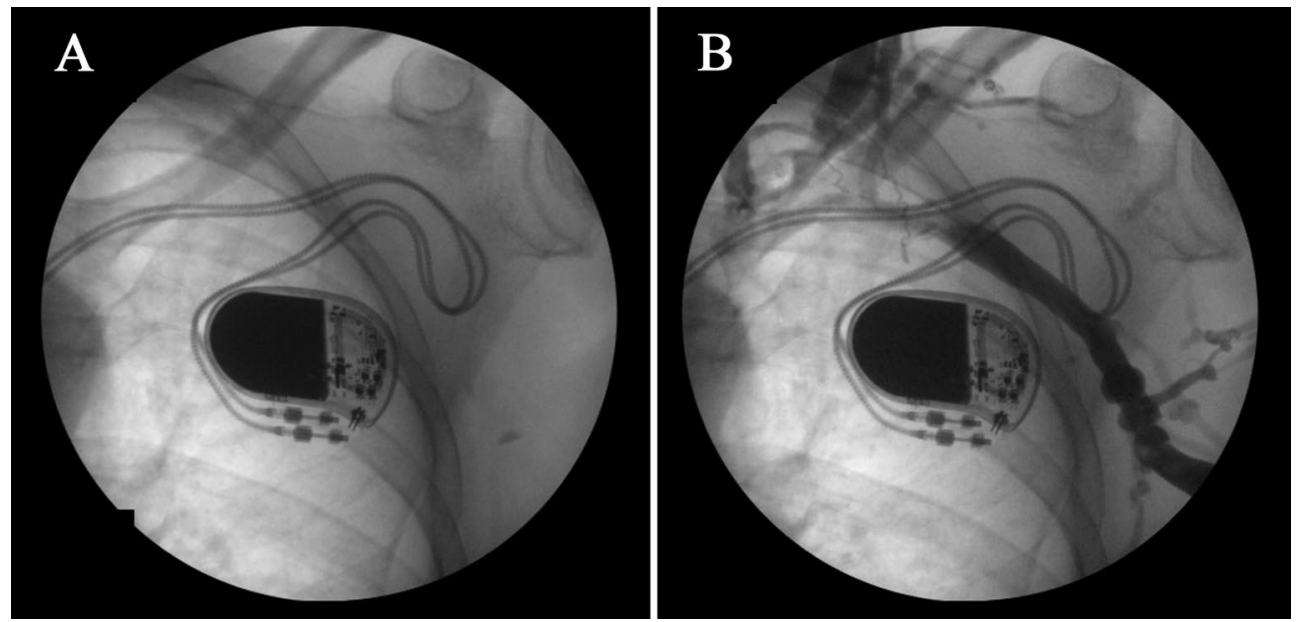

Figure 3. Status post pacemaker reposition towards the anterior median line $(\mathbf{A})$. Normalized flow of contrast through the previously compressed axillary vein (B) (see Fig. IB)

flaccid adjacent tissues or tissue involution due to considerable weight loss). CIED migration towards the armpit may result in persistent or intermittent compression of the anatomical structures typically found in this region, such as the axillary vein (along its course underneath the pectoralis minor muscle) or branches of the axillary nerve $[4,5]$. Such compression may produce symptoms of varying character and severity, such as edema, pain, numbness, paresthesia, or cyanosis in the ipsilateral upper limb.

Patients presenting with such symptoms require differential diagnosis, as they may have potentially coexisting diseases that produce similar symptoms but require different therapeutic strategies. Such diseases include post-thrombotic lesions, Paget-Schroetter syndrome, and Pectoralis Minor Syndrome [I, 2, 6].

Slight shifts in the position of an implanted device are not uncommon with CIED implantation procedures, and usually do not require surgical intervention, as they typically have no adverse clinical consequences. Symptoms such as those presented here developed in one out of all the patients who underwent a CIED implantation procedure in our center between the years 2012 and 2020.

\section{References}

I. Riezebos RK, Schroeder-Tanka J, de Voogt WG. Occlusion of the proximal subclavian vein complicating pacemaker lead implantation. Europace. 2006; 8(I): 42-43, doi: 10.1093/europace/euj002, indexed in Pubmed: 16627407.

2. Sharma H, Tiwari A. Recurrent Upper Extremity Thrombosis Associated with Overactivity: A Case of Delayed Diagnosis of Paget-Schroetter Syndrome. Case Rep Vasc Med. 2017; 2017: 8764903, doi: 10.1155/2017/8764903, indexed in Pubmed: 28775908

3. Sanders RJ, Annest SJ. Pectoralis Minor Syndrome: Subclavicular Brachial Plexus Compression. Diagnostics (Basel). 2017; 7(3), doi: 10.3390/diagnostics7030046, indexed in Pubmed: 28788065 .

4. Rizniotopoulou P, Apostolou CE, Fountoukis TN, et al. Pectoralis minor syndrome: Report of two cases and review. Hellenic Journal of Surgery. 2017; 89(3-4): 149-152, doi: 10.1007/ s|3|26-017-0402-6.

5. Yang HJ, Gil YC, Jin JD, et al. Novel findings of the anatomy and variations of the axillary vein and its tributaries. Clin Anat. 2012; 25(7): 893-902, doi: 10.1002/ca.22086, indexed in Pubmed: 22623347.

6. Abdallah M, Wehbe MR, Elias E, et al. Pectoralis Minor Syndrome: Case Presentation and Review of the Literature. Case Rep Surg. 2016; 2016: 8456064, doi: 10.1155/2016/8456064, indexed in Pubmed: 27429830. 